\title{
DIFFERENT TYPES OF CONNECTED SPACES
}

\author{
M. Sattanathan* and Jomon K. Sebastian**
}

\section{ABSTRACT}

This paper deals with the various forms of open sets and their relations. The relation is represented by a diagram. The relation is same for the closed sets also. The main part defines different types of connectedness and their relations. It is notable that openness and connectedness are in opposite order. Some results on Q-sets are also included here.

\section{Introduction}

Let $X$ be a topological space and let $A$ be a subset of $X$. $c l(A)$ and int $(A)$ denote the closure and interior of $A$ in $X$ respectively. Here $R$ denotes the set of all real numbers. We recall the following definitions from [1] and [2].

\section{Definition 1.1:}

A subset $A$ of a space $(X, \bullet)$ is called a semi-open set if $A \subseteq C l(i n t(A))$ and a semiclosed set if int $(c)(A)) \subseteq A$.

* Department of Mathematics, St. Joseph's College (Autonomous), Tiruchirappalli-620 002

** Department of Mathematics, St. Joseph's College (Autonomous), Tiruchirappalli-620 002 


\section{Definition 1.2:}

A subset $A$ of space $(X, \bullet)$ is called a pre-open set if $A \subseteq \operatorname{int}(c)(A))$ and a pre-closed set if $\mathrm{cl}(\mathrm{int}(\mathrm{A})) \subseteq \mathrm{A}$.

\section{Definition 1.3:}

A subset $A$ of space $(X, \cdot)$ is called an a-open set if $A \subseteq \operatorname{int}(\operatorname{cl}($ int $(A)))$ and an $\alpha$-closed set if $c \mid(\operatorname{int}(c \mid(A))) \subseteq A$.

\section{Definition 1.4:}

A subset $A$ of a space $(X, \bullet)$ is called a b-open set (semi-pre open set) if $A \subseteq$ $c l($ int $(c l(A)))$ and a b-closed set (semi-pre closed set) if int(cl(int $(A))) \subseteq A$.

\section{Theorem 1.5:}

The complement of a semi-open set is a semi-closed set .

\section{Proof:}

Let $A$ be a semi-open set.

$$
\begin{aligned}
& \therefore A \subseteq \mathrm{cl}(\operatorname{int}(A)) \\
& \Rightarrow\left[d\{\operatorname{int}(A)]^{c} \subseteq A^{c}\right. \\
& \Rightarrow \operatorname{int}(\operatorname{int} A)^{c} \subseteq A^{c} \\
& \Rightarrow \operatorname{int}\left(c \mid A^{c}\right) \subseteq A^{c}
\end{aligned}
$$

Hence $A^{c}$ is a semi-closed set.

\section{Remark 1.6:}

Similar to theorem 1.5, the complement of a pre-open(resp. $\beta$-open, $\alpha$-open) set is a pre-closed (resp.b-closed, a-closed)set.

\section{Remark 1.7:}

All open sets are semi-open, pre-open, $\alpha$-open, and $\beta$-open sets. 


\section{Main Results}

\section{Theorem 2.1 :}

Any a-open set is a semi-open set.

\section{Proof:}

Let $\mathrm{A}$ be an a-open set.

$\therefore A \subseteq \operatorname{int}(\mathrm{cl}(\operatorname{int}(A))) \subseteq \mathrm{cl}(\operatorname{int}(A))$

$\Rightarrow A i ́ d(\operatorname{int}(A))$

$\Rightarrow A$ is semi-open.

\section{Remark 2.2:}

Converse of theorem 2.1 is not true.

In $R$, the $\operatorname{set} A=[0,1)$ is semi-open but not $\alpha$-open.

\section{Theorem 2.3:}

Any $\alpha$-open set is a pre-open set.

\section{Proof:}

Let $A$ be an $\alpha$-open set. Then $A \subseteq \operatorname{int}(c l(\operatorname{int}(A)))$

We know that $\operatorname{int}(A) \subseteq A$.

$\therefore \mathrm{cl}(\operatorname{int}(A)) \subseteq \mathrm{cl}(A)$.

$A \subseteq \operatorname{int}(c \mid(\operatorname{int}(A))) \subseteq \operatorname{int}(c \mid(A))$ by $(T)$.

$\therefore A \subseteq \operatorname{int}(c \mid(A))$.

$\therefore A$ is pre-open. 


\section{Remark 2.4:}

Converse of theorem 2.3 is not true.

In $R, Q=$ the set of all rational numbers, is a pre-open set but not $\alpha$-open.

\section{Theorem 2.5:}

Any semi-open set is a $\beta$-open set.

\section{Proof:}

Let $A$ be a semi-open set.

$\therefore A \subseteq \mathrm{d}(\operatorname{int}(A))$

Always $A \subseteq c$ (A).Hence

$\operatorname{int} A \subseteq \operatorname{int} d(A)$.

$\therefore A \subseteq \mathrm{cl}(\operatorname{int}(A)) \subseteq \mathrm{d}(\operatorname{int}(\mathrm{cl}(\mathrm{A})))$ by $(1)$

$\therefore A \subseteq c \mid(\operatorname{int}(\mathrm{cl}(\mathrm{A}))$ and so

$A$ is a $\beta$-open set.

\section{Remark 2.6:}

Converse of theorem 2.5 is not true.

In $R, Q=$ set of all rational numbers, is $\beta$ - open but not semi-open.

\section{Theorem 2.7:}

Any pre-open set is $\alpha \beta$-open set.

\section{Proof:}

Let $A$ be a pre-open set.

$\therefore A \subseteq \operatorname{int}(\mathrm{Cl}(\mathrm{A}))$ and so 
$c \mid(A) \subseteq \mathrm{cl}(\operatorname{int}(\mathrm{cl}(\mathrm{A})))$. Thus

$A \subseteq \mathrm{cl}(A) \subseteq \mathrm{cl}(\operatorname{int}(\mathrm{cl}(\mathrm{A})))$.

$\therefore A \subseteq \mathrm{cl}(\operatorname{int}(\mathrm{cl}(\mathrm{A})))$.

$\therefore A$ is a $\beta$-open set.

\section{Remark 2.8:}

Converse of theorem 2.7 is not true.

$\ln R$, the set $A=[0,1)$ is $\beta$-open but not pre-open.

\section{Remark 2.9:}

$\alpha$-open

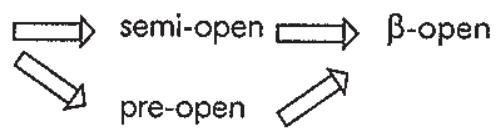

Similarly,

a-closed $\Longrightarrow$ semi-closed $\Longrightarrow$ pre-closed

\section{Definition 2.10:}

Let $X$ be a topological space. $X$ is said to be semi-connected if $X$ cannot be written as the union of two nonempty disjoint semi-open subsets of $X$.

\section{Definition 2.11:}

Let $X$ be a topological space. $X$ is said to be pre-connected if $X$ cannot be written as the union of two nonempty disjoint pre-open subsets of $X$.

\section{Definition 2.12:}

Let $X$ be a topological space. $X$ is said to be a-connected if $X$ cannot be written as the union of two non empty disjoint a-open subsets of $X$. 


\section{Definition 2.13:}

Let $X$ be a topological space. $X$ is said to be $\beta$-connected if $X$ cannot be written as the union of two non empty disjoint $b$-open subsets of $X$.

The complement of a-open (resp. semi-open, pre-open, b-open) is a-closed (resp. semi-closed, pre-closed, b-closed). So we have the following results.

\section{Results 2.14:}

1. Let $\mathrm{X}$ be a topological space. $\mathrm{X}$ is semi-connected (resp. pre-connected, $\alpha$-connected, $\beta$-connected) if $X$ cannot be written as the union of two nonempty disjoint semi-closed (resp. pre-closed, $\alpha$-closed , $\beta$-closed) subsets of $X$.

2. Let $X$ be a topological space. $X$ is semi-connected (resp. pre-connected, $\alpha$-connected, $\beta$-connected) if the only subsets of $X$ that are both semi-open (resp. pre-open, $\alpha$-open, $\beta$-open) and semi-closed (resp. pre-closed, $\alpha$-closed, $\beta$-closed I are $\mathrm{X}$ and $f$ only.

\section{Theorem 2.15:}

Every $\beta$-connected space is semi-connected.

\section{Proof:}

Assume $X$ is $\beta$-connected.

Claim: $X$ is semi-connected.

Suppose $X$ is not semi-connected.

Then $\exists$ a non-empty subset $A$ of $X$ which is both semi-open and semi-closed.

We know that semi-open $\Rightarrow \beta$-open and semi-closed $\Rightarrow \beta$-closed.

Hence $A$ is both $\beta$-open and $\beta$-closed which is a contradiction to the fact that $X$ is $\beta$-connected.

\section{Remark 2.16:}

The converse of theorem 2.15 is not true. 
For example,

$$
\begin{aligned}
\text { let } X & =\{a, b, c\} \\
\bullet & =\{\{a, c\}, X, \phi\} .
\end{aligned}
$$

The open sets are $\{a, c\}, X, \phi$.

Closed sets are $\{b\}, X, \phi$.

Semi-open sets are $\{a, c\}, X, \phi$.

$b$-open sets are $\{a, c\}, X, \phi,\{a, b\},\{b, c\},\{a\},\{c\}$.

$X=\{a\} \cup\{b, c\}$ gives a separation of $X$ into a non-emply disjoint union of $\beta$ open sets and so $X$ is semi-connected but not $\beta$-connected.

\section{Theorem 2.17:}

Every semi-connected space is $\alpha$-connected.

\section{Proof:}

Assume $X$ is semi-connected.

Claim: $X$ is $\alpha$-connected.

Suppose $X$ is not $\alpha$-connected.

Then $\exists$ a non-empty subset $A$ of $X$ which is both $\alpha$-open and $\alpha$ -

closed. We know that a-open $\Rightarrow$ semi-open and $\alpha$-closed $\Rightarrow$ semi-

closed. Hence $A$ is both semi-open and semi-closed

which is a contradiction to the fact that $X$ is semi-connected.

\section{Remark 2.18:}

The converse of theorem 2.17 is not true.

For example,

let $X=\{a, b, c\}$

$$
\text { - }=\{\{a\},\{c\},\{a, c\}, X, \phi\}
$$

The open sets are $\{a\},\{c\},\{a, c\}, X, \phi$.

Closed sets are $\{b, c\},\{a, b\},\{b\}, X, \phi$. 
semi-open sets are $\{a\},\{c\},\{a, c\},\{b, c\},\{a, b\},\{b\}, X, \phi$.

$\alpha$-open sets are $\{a\},\{c\},\{a, c\}, X, \phi$.

$X=\{a\} \cup\{b, c\}$, gives a separation as $X$ into a non-empty disjoint union of semi-open sets and so $X$ is $\alpha$-connected but not semi-connected.

\section{Theorem 2.19:}

Every $\beta$-connected space is pre-connected.

\section{Proof:}

Assume $X$ is $\beta$-connected.

Claim: $\mathrm{X}$ is pre-connected.

Suppose $\mathrm{X}$ is not pre-connected.

Then $\exists$ a non-empty subset $A$ of $X$ which is both pre-open and preclosed.

We know that pre-open $\Rightarrow \beta$-open and pre-closed $\Rightarrow \beta$-closed.

Hence $A$ is both $\beta$-open and $\beta$-closed

which is a contradiction to the fact that $X$ is $\beta$-connected.

\section{Remark 2.20:}

The converse of theorem 2.19 is not true.

For example, consider the topological space as in 2.18. Then

pre-open sets are $\{a\},\{c\},\{a, c\}, X, \phi$.

b-open sets are $\{a\},\{c\},\{a, c\},\{a, b\},\{b, c\}, X, \phi$.

$X=\{a\} \cup\{b, c\}$, gives a separation of $X$ into a non-empty disjoint union of $\beta$-opensets and so $X$ is pre-connected but not $b$-connected.

\section{Theorem 2.21:}

Every pre-connected space is a-connected. 


\section{Proof:}

Assume $X$ is pre-connected.

Cloim: $X$ is pre-connected.

Suppose $X$ is not $\alpha$-connected.

Then $\exists$ a non-empty subset $A$ of $X$ which is both $\alpha$-open and $\alpha$-closed.

We know that $\alpha$-open $\Rightarrow$ pre-open and $\alpha$-closed $\Rightarrow$ pre-closed.

Hence $A$ is both pre-open and pre-closed which is a contradiction to the fact that $X$ is pre-connected.

\section{Remark 2.22:}

The converse of theorem 2.21 is not true. For example, consider the topological space defined on 2.16. Then the $a$-open sets are $\{a, c\}, X, \phi$.

pre-open sets are $\{a\},\{c\},\{a, b\},\{b, c\},\{a, c\}, X, \phi$.

$X=\{a\} \cup\{b, c\}$, gives a separation as $X$ into a non-empty disjoint union of preopen sets and so $X$ is a-connected but not pre-connected.

Thus we have

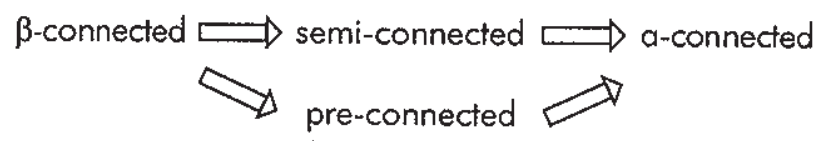

\section{Remark 2.23:}

By remark 1.7, all semi-connected, pre-connected, $\alpha$-connected, $\beta$-connected spaces are connected.

\section{Remark 2.24:}

Consider $R$ with usual topology,

(i) $\quad[a, b]$ is both semi-open and semi-closed and so $R$ is connected but not semi-connected.

(ii) $Q$ is both pre-open and pre-closed and so $R$ is connected but not pre-connected. 
(iii) $Q$ is both $\beta$-open and $\beta$-closed and so $R$ is connected but not $\beta$-connected.

\section{Definition 2.25:}

$A$ set $A$ is said to be $Q$-set if int $(c l(A))=\operatorname{cl}(\operatorname{int}(A))$.

\section{Theorem 2.26:}

dense.

In a connected space every $Q$-set is either nowhere dense or everywhere

\section{Proof:}

Let $X$ be a connected space.

Let $A$ be a $Q$-set in $X$.

$\therefore \operatorname{int}(c \mid(A))=\operatorname{cl}(\operatorname{int}(A))$.

Take $B=\operatorname{int}(c)(A))=\operatorname{cl}(\operatorname{int}(A))$.

Hence $B$ is both open and closed set in $X$.

Since $X$ is connected either $B=\phi$ or $B=X$.

If $B=X$, then $c \mid(\operatorname{int}(A))=X$.

Since int $(A) \subseteq A$,

$c l(i n t(A)) \subseteq c l(A)$ and so

$X \subseteq \mathrm{cl}(A)$.

$\therefore \mathrm{Cl}(\mathrm{A})=\mathrm{X}$.

Hence $A$ is everywhere dense.

If $B=\phi$, then

$\operatorname{int}(\mathrm{cl}(\mathrm{A}))=\phi$.

Hence $A$ is nowhere dense.

\section{Result 2.27:}

If $a Q$-set is $\beta$-open then it is necessarily semi-open. 


\section{Proof:}

$$
\begin{aligned}
& \text { Let } A \text { be } a Q \text {-set. } \\
& \therefore \operatorname{int}(c \mid(A))=c l(\operatorname{int}(A))
\end{aligned}
$$

Since $A$ is $\beta$-open, $A \subseteq c l(i n t(c)(A)))$

i.e. $A \subseteq \mathrm{cl}(\mathrm{c})(\mathrm{int}(A))) \quad$ by $(1)$

i.e. $A \subseteq \mathrm{cl}($ int $(A))$

Hence $A$ is semi-open.

\section{Result 2.28:}

If a $Q$-set is $\alpha$-open then it is necessarily pre-open.

\section{Proof:}

Let $A$ be a $Q$-set.

$$
\therefore \operatorname{int}(\mathrm{cl}(\mathrm{A}))=\mathrm{dl}(\operatorname{int}(\mathrm{A}))
$$

Since $A$ is $\alpha$-open, $A 1 \operatorname{int}(c)(\operatorname{int}(A)))$

i.e. $A \subseteq \operatorname{int}(\operatorname{int}(c)(A)))$ by (1)

i.e. $A \subseteq \operatorname{int}(c \mid(A))$

Hence $A$ is pre-open.

\section{Open problem:}

(i) Construct a topological space which is connected but not a-connected.

\section{References:}

1. Chandrasekhara Rao K and Dhano Balan A P: Some Functions on a Topological Space-ll, The Mathematical Education, Vol.35, No. 1, March 2001.

2. Veerakumar MKRS: Between Semi-Closed Sets And Semi-Pre-Closed Sets, htto:ll www, angelfire,com/ca5/veerakumar/psiclosed.htm 